\title{
Pathological Role of Tonsillar B Cells in IgA Nephropathy
}

\author{
Yusuke Suzuki, Hitoshi Suzuki, Junichiro Nakata, Daisuke Sato, Tadahiro Kajiyama, \\ Tomonari Watanabe, and Yasuhiko Tomino
}

Division of Nephrology, Department of Internal Medicine, Juntendo University Faculty of Medicine, Hongo 2-1-1, Bunkyo-ku, Tokyo 113-8421, Japan

Correspondence should be addressed to Yusuke Suzuki, yusuke@juntendo.ac.jp

Received 30 January 2011; Accepted 17 May 2011

Academic Editor: K. Blaser

Copyright () 2011 Yusuke Suzuki et al. This is an open access article distributed under the Creative Commons Attribution License, which permits unrestricted use, distribution, and reproduction in any medium, provided the original work is properly cited.

\begin{abstract}
Although impaired immune regulation along the mucosa-bone marrow axis has been postulated to play an important role, the pathogenesis of IgA nephropathy (IgAN) is unknown; thus, no disease-specific therapy for this disease exists. The therapeutic efficacy of tonsillectomy or tonsillectomy in combination with steroid pulse therapy for IgAN has been discussed. Although randomized control trials for these therapies are ongoing in Japan, the scientific rationale for these therapies remains obscure. It is now widely accepted that abnormally glycosylated IgAl and its related immune complex (IC) are probably key molecules for the pathogenesis, and are thus considered possible noninvasive biomarkers for this disease. Emerging evidence indicates that $\mathrm{B}$ cells in mucosal infections, particularly in tonsillitis, may produce the nephritogenic IgA. In this paper, we briefly summarize characteristics of the nephritogenic IgA/IgA IC, responsible B cells, and underlying mechanisms. This clinical and experimental information may provide important clues for a therapeutic rationale.
\end{abstract}

\section{Introduction}

IgA nephropathy (IgAN) is the most common form of glomerulonephritis (GN) globally, accounting for $25 \%-50 \%$ of primary GN patients [1]. Long-term follow-up studies have shown that up to $25 \%-30 \%$ of IgAN patients progress to end-stage kidney disease within 20-25 years [2]. However, the pathogenesis of IgAN remains unclear, and consequently, no disease-specific therapy for IgAN exists.

The recurrence of IgA deposition in renal allografts [3] and the disappearance of IgA deposits from renal allografts taken from donors with undiagnosed IgAN $[4,5]$ reinforce the importance of systemic abnormalities of the IgA immune system in IgAN, arguing against IgAN being a disease limited to intrinsic renal abnormalities. Several clinical studies have identified the importance of IgA or IgA-IC deposition as a fundamental causative factor in IgAN [6]. The observed clinicopathological heterogeneity may, at least in part, be dependent on the characteristics of the deposited IgA-IC itself or changes in the IgA immune system, including sites of IgA synthesis and stimulation and regulation of immunecompetent cells involved in the production of IgA [7].
On the other hand, the episodic macrohematuria, coinciding with mucosal infection such as tonsillitis and pharyngitis [8] or an abnormal response to mucosal vaccination in IgAN patients $[9,10]$, indicates that dysregulation of the mucosal immune system may play an important role in the pathogenesis of IgAN [6]. In addition, tonsillectomy is effective in long-term renal survival in IgAN patients [11]. Some Japanese studies have recently reported that tonsillectomy in combination with steroid pulse therapy can be a more effective therapy for IgAN than tonsillectomy alone [12-14].

However, the therapeutic validity of tonsillectomy and the indication for tonsillectomy for IgAN are controversial [15-17], even in Japan. Although tonsillectomy in certain patients can be an effective therapy, $7 \%-10 \%$ of IgAN patients show spontaneous clinical remission [18]. Therefore, a rationale and reasonable clinical markers are needed for indication of this therapy. Recent studies show that predictive factors for resistance to tonsillectomy in combination with steroid pulse therapy are age of onset, severity of proteinuria and hematuria, and pathological grade [19]. Although there is an ongoing randomized control trial evaluating the effect of tonsillectomy on this 
disease, mainly by the Special Study Group on Progressive Glomerular Disease of the Ministry of Health, Labor, and Welfare of Japan and the Japanese Society of Nephrology, the results are not yet available.

Many reports demonstrate that tonsillectomy is an effective therapy for dermatological diseases such as pustulosis palmaris et plantaris and psoriasis, sternocostoclavicular hyperostosis, and rheumatoid arthritis [20-23]; the rationale for this effect is also unknown. In contrast, elucidation of the rationale in IgAN may provide conclusive clues for the pathogenesis of not only IgAN but also the so-called "tonsillar focal infectious diseases." To assess that rationale, we briefly summarize the characteristics of nephritogenic IgA and the $\mathrm{B}$ cells responsible for producing the nephritogenic IgA.

\section{Generation of Nephritogenic IgA in the Mucosa-Bone Marrow (BM) Axis in IgAN}

High levels of higher molecular forms of IgA are present in the serum of IgAN patients [2, 6, 7]. In addition, it is generally accepted that IgA deposits in glomerular mesangium primarily consist of polymeric forms of IgA1 including IC [2, $6,7,24]$. Large numbers of polymeric IgA- (pIgA-) positive plasma cells are found in $\mathrm{BM}$ in $\operatorname{IgAN}[6,7,25]$. Moreover, $\mathrm{BM}$ transplantation (BMT) in leukemia and IgAN patients has resulted in curing not only of leukemia but also of IgAN $[26,27]$, suggesting that overproduction of nephritogenic pIgA1 in IgAN seems to be partly based in systemic immune sites, such as BM. Furthermore, mucosal vaccination results in impaired mucosal IgA responses in IgAN whereas systemic antigen challenge results in increased titers of circulating pIgA1 antibodies with normal levels in mucosal secretions $[28,29]$. In addition, not only $\operatorname{IgA}^{+}$cells but also polymeric IgA are increasingly produced in the mucosa of IgAN patients [30-32]. On the other hand, there is a report demonstrating that there is a reduction in polymeric IgA producing cells in duodenum [33]. Therefore, the crosstalk between the mucosa and BM should be carefully discussed. Furthermore, we should remember that the tonsil has distinctive immunophenotypic characteristics and immune status including anatomical location, and population, and/or distribution of lymphocytes from the rest of the mucosal immune system.

In a series of studies published almost 20 years ago, Van Es et al. identified impaired IgA immune responses in the mucosa-BM axis in IgAN $[25,29,34,35]$. In the last decade, clinical and experimental studies have revealed continued trafficking of antigen-specific lymphocytes and antigenpresenting cells between the mucosa and BM in humans $[6,36]$. The patterns of integrins and chemokine receptors in these lymphocytes, including memory B cells and IgA plasma cells, are slightly different and depend on the site of induction. This migration is directed by the local synthesis of specific chemokines and appropriate adhesion/homingreceptor engagement. There is increasing recognition for the presence of a mucosa-BM axis in humans, and abnormalities in this estimated axis may play an important role in the development of IgAN [6].

\section{Characteristics of Abnormally Glycosylated IgA in IgAN}

It is now apparent that serum levels of galactose-deficient IgA1 (GdIgA1), mainly in IC forms, are often elevated in IgAN patients [37-39]. Moldoveanu et al. recently demonstrated, using a lectin-binding assay, that Caucasian IgAN patients have increased levels of serum GdIgA1 [37]. The mesangial IgA deposits also display abnormal $O$ glycosylation $[40,41]$ in IgAN. It is known that underglycosylated IgA tends to be aggregated, indicating that the polymeric formation of IgA1 may be, at least in part, based on the aberrant glycosylation of IgA1 [42-44]. Furthermore, pIgA has more capacity to activate mesangial cells than monomeric IgA [45-49]. Accordingly, the involvement of GdIgA in the pathogenesis of IgAN has been discussed $[2,50,51]$. A recent study has shown that IgA1 secreted by Epstein-Barr virus-immortalized B cells from peripheral blood of IgAN patients was mostly polymeric, and had galactose-deficient sialylated $O$-glycans due to decreased $\beta 1$, 3 -galactosyltransferase (core $1 \beta$ GalT) activity, and increased $N$-acetylgalactosamine-specific $\alpha 2,6$-sialyltransferase activity [38]. On the other hand, IgA1 produced by tonsillar lymphocytes is also abnormally glycosylated in IgAN patients [52-55]. These clinical findings taken together further support the idea that the estimated mucosa-BM axis is the location where B cells producing the nephritogenic GdIgA1 may traffic between tonsil and other organs via peripheral blood in human IgAN [36].

Humans have two isotypes of IgA: IgA1 and IgA2. IgA1 contains $\mathrm{O}$-glycosylation sites but IgA2 or murine IgA do not [56]. Thus, the murine model does not include the aberrant $O$-glycosylation involved in the human IgAN. However, recent studies suggest that aberrant glycosylation of $N$-glycans may be involved in the pathogenesis of murine IgAN [57, 58], suggesting that aberrant modifications of carbohydrates of serum IgA are involved in the development of not only human but also murine IgAN, whether the carbohydrates are $\mathrm{O}$-glycans or $\mathrm{N}$-glycans.

Renal biopsy and subsequent immunohistochemical analysis of the renal tissue remains the gold standard for diagnosing IgAN or evaluating the activity of acute lesions of this disease; however, new sensitive, and reasonably specific, non-invasive tests are emerging and may provide another diagnostic approach. Our recent study showed that reduction of serum GdIgA1 after tonsillectomy was associated with improvement of hematuria in certain IgAN patients (our unpublished data). Since the primary abnormal clinical manifestation in IgAN is recurring bouts of hematuria with or without associated proteinuria [59], elevated circulating levels of GdIgA1 is one of the most promising new tests for diagnosis or activity of IgAN [37, 40, 41, 52-54].

However, Gharavi et al. have recently reported that GdIgA1 levels were increased in $78 \%$ of sporadic IgAN patients and in $25 \%$ of their blood relatives, although the majority of relatives with abnormally glycosylated IgA1 were asymptomatic [60]. This finding suggests that, in certain cases, additional cofactors are required for development of IgAN. In a recent publication, Suzuki and coworkers 
described the characteristics of IgG autoantibodies to abnormally glycosylated IgA1 secreted by immobilized B cells derived from sporadic IgAN patients [61]. The serum levels of these IgG autoantibodies correlated closely with the degree of proteinuria, suggesting that IC formation of aberrantly glycosylated IgA and glycan-specific IgG antibodies and subsequent enlargement of their molecular mass may be an additional cofactor required for full development of the disease $[62,63]$. In this regard, it is noteworthy that the serum levels of IgA-IgG2a IC, but not of IgA, were shown to correlate closely with the severity of glomerular lesions in IgAN-prone mice [64]. In addition to the aberrant glycosylation of IgA, similar mechanisms may underlie the progression of both human and murine IgAN.

\section{Mucosal Encounter of Nephritogenic Exogenous Antigens in IgAN}

The association of episodic macroscopic hematuria with mucosal infections in IgAN is suggestive of changes to the mucosal immune system in this disease, which may include changes in antigen handling [6]. The results of immunization studies in IgAN patients support that notion. Mucosal immunization with neoantigen results in impaired mucosal and systemic IgA responses but normal IgG and IgM responses $[9,10]$, suggesting that in IgAN there is mucosal hyporesponsiveness to mucosal neoantigens. In contrast, systemic and mucosal immunization with recall antigens results in exaggerated systemic IgA responses with increased and prolonged production of specific IgA [11, 12, 28, 65]. These results suggest that IgAN patients respond excessively to recall antigens [6].

Common microbial and food or food-borne antigens may also play a role in this process. Emancipator et al. [66] demonstrated a pathogenic relationship between prolonged mucosal antigenic exposure, formation of circulating IgAIC, and the development of GN. Those authors orally immunized Balb/c mice with protein antigens and found a significant increase in specific IgA-producing plasma cells in the lamina propria of bronchial and intestinal mucosae coupled with a rise in circulating antigen-specific $\operatorname{IgA}$ and mesangial deposits of IgA and J chain. Coppo et al. [67] have argued that altered mucosal processing of food antigens such as gliadin, a lectin present in gluten, might be involved in the induction of this disease. High serum levels of IgA antigliadin have been reported in IgAN patients [68-70]. Coppo et al. [71] have also demonstrated that mice orally immunized with gliadin or ovalbumin developed glomerular injury with intense glomerular IgA deposition, including antigliadin IgA antibodies. In addition to intrinsic food antigens, foodborne microbial contaminants may also provide an antigenic stimulus in IgAN. Pestka et al. have demonstrated that mice fed meals contaminated with deoxynivalenol developed increased levels of serum IgA, circulating IgA-IC, mesangial IgA deposition, and hematuria, all clinical features of human IgAN [72-75]. However, in human IgAN, there are many studies indicating that the levels of antibodies to food antigens as well as microbial antigens are not any different from normal individuals $[76,77]$. Therefore, we have to carefully think differences in immune responses to mucosally encountered antigens, such as quality of antibodies or IC between human and mouse.

\section{Tonsillar B Cells in the Generation of Nephritogenic IgA in IgAN}

Considered together, these studies suggest that mucosal encounter with exogenous antigens derived from fungi, bacteria, and viruses could play a key role in the development of IgAN. On the other hand, one can speculate that there is not a single infectious or food antigen which has been uniformly and convincingly associated with the development of IgAN. It remains unclear, however, precisely how all these microberelated antigens interact with the IgA immune system to trigger disease. It is actually difficult to assess this question using human samples alone. Therefore, we used animal models. We were able to establish a spontaneous IgAN-prone murine model $[6,78]$ and adopted it for our purpose. We found that human and murine IgAN are regulated, at least partly, by the same genes in addition to the same phenotype of renal damage [78]. Growing evidence from studies of innate immunity may provide a clue. Toll-like receptors (TLRs) are a family of pathogen recognition molecules that discriminate self from nonself (pathogens) and activate suitable defense mechanisms [79]. TLRs on antigen-presenting cells also initiate and modulate adaptive immunity during infection [80]. To assess these questions in reference to IgAN, we examined the pathological roles of TLRs in this IgAN-prone murine model [81]. An association study using an IgANprone murine model showed that the progression of murine IgAN was linked to signaling molecules of the TLR myeloid differential protein 88 (MyD88) [81]. We also examined the relationship between the TLR mRNA expression level in splenocytes and disease activity, and found that the severity of glomerular injury in this model was clearly linked only to the degree of MyD88 and TLR9 expression in splenocytes [81]. TLR9 recognizes microbial unmethylated DNA and is expressed mainly by B cells and dendritic cells (DC) in mucosa [82-84]. In fact, intranasal immunization with CpG oligodeoxynucleotide (CpG-ODN), which is known as a ligand for TLR9, aggravated glomerular damage with elevation of the serum IgA level [81], confirming that TLR, and in particular TLR9, is involved in the pathogenesis of murine IgAN.

We have also confirmed that transfer of BM from the IgAN-prone to wildtype control mice results in the development of IgAN [64]. In contrast, BM transfer from wildtype normal mice to the onset-prone mice abrogates glomerular injury and mesangial deposition. These findings suggest that BM may be a reservoir of memory cells capable of synthesizing IgA with a propensity for mesangial deposition and triggering of GN in murine IgAN, as in human IgAN $[26,27]$.

Are these cells responsible for the nephritogenic IgA production reserved only in BM? To approach the possibility that these responsible cells are disseminated to other lymphoid tissues, we transferred whole spleen cells from the IgAN-prone mice into subacute combined immunodeficient (SCID) mice. BMT as well as cell transfer could reconstitute 
the murine IgAN in SCID mice, suggesting that the responsible cells may be also localized in systemic lymphoid organs (Nakata et al., paper in preparation). Adoptive transfer analyses in the same study showed that $\mathrm{CD} 19^{+}$cells are necessary for the development of murine IgAN. CD19 is expressed on follicular DC and B cells. Since CD19 ${ }^{+}$cell transfer induced glomerular IgA deposition with elevation of serum IgA even in SCID mice lacking $\mathrm{T}$ and $\mathrm{B}$ cells, responsible $\mathrm{CD} 19^{+}$cells in this disease may be mainly $\mathrm{B}$ cells.

CD19 is present on B cells from earliest recognizable Blineage cells during development to B cell blast but is lost on maturation to plasma cells [85]. On the other hand, CD 138 is known to be a cell surface marker for plasma cells [86]. Our recent study also showed that CD138 ${ }^{+}$cells are seen in BM of reconstituted IgAN mice whereas those from spleen did not induce glomerular IgA deposition or glomerular lesions in Balb/c mice. Pre-B or immature B cells in murine BM also express CD138; the cells lose their capacity for CD138 expression after emigrating from BM, but re-express it during the final maturation to plasma cells in peripheral lymphoid tissues or spleen. Therefore, the responsible cells may be $\mathrm{CD}_{1} 9^{+} \mathrm{B}$ cells but not mature CD $138^{+}$plasma cells, at least in murine IgAN. Furthermore, reconstitution of IgAN by cell transfer of spleen cells depleted of the $\mathrm{CD}^{+} 0^{+}$pan $\mathrm{T}$ cell in both murine strains and cell transfer of CD19+ cells in SCID mice indicates that the responsible $\mathrm{B}$ cells link to the nephritogenic IgA in a Tcell-independent manner. Our recent experimental study using alymphoplasic (aly/aly) mice [87] further supports this notion. Aly/aly mice have no secondary lymphoid tissues or germinal center (GC) [88]. However, BMT from our IgANprone mice also reconstituted glomerular IgA deposition and glomerular lesions in aly/aly recipient mice [87]. This finding suggests that somatic hypermutation in GC may not be required for nephritogenic IgA production, at least in murine IgAN. Accordingly, B cells related to the nephritogenic IgA may mature in GC-independent and T-cell-independent manners. Therefore, B-DC interaction should be carefully evaluated. At the very least, both cells express TLR9 and are key players in mucosal immunity.

Does TLR9 contribute to progression of human IgAN? Our previous study demonstrated that two genotypes of TLR9 have a strong association with the progression of IgAN, indicating the involvement of TLR9 also in the pathogenesis of human IgAN [81]. We demonstrated that the CC/CT genotype in rs352139 and TT genotype in rs352140 could be risk factors for the progression of IgAN. As mentioned previously, the tonsil can be a major mucosal site in the pathogenesis of human IgAN. Human tonsils contain relatively few IgA-producing cells, which in vitro secrete predominantly monomeric and polymeric IgA1. The contribution of tonsillar IgA1-producing cells to the total pool of IgA produced in the human body must be minimal considering the absolute number of cells in tonsils versus the mucosal tissues such as gut, bone marrow, lymph nodes, and spleen. However, most IgAN patients indeed showed a decrease in serum IgA after tonsillectomy, in association with improvement of clinical manifestations [89-91]. Moreover, tonsillar B cells in IgAN patients produced more GdIgA1 and pIgA than those of chronic tonsillitis or sleep apnea syndrome [52]. Further, tonsillar CD19+ $\mathrm{B}$ cells in IgAN also showed downregulation of core $1 \beta \mathrm{GalT}$ expression, which is inversely correlated with estimated glomerular filtration rate [55].

Our recent study demonstrates that some IgAN patients exhibited relatively high expression of tonsillar TLR9 mRNA (the TLR9 high group) and showed an earlier, more complete clinical remission than those with low TLR9 expression [92]. Patients whose serum IgA decreased more than the average of enrolled IgAN patients after tonsillectomy (Large $\Delta \operatorname{IgA}$ ) showed higher cumulative remission rates of proteinuria than did the others (Small $\Delta \operatorname{IgA}$ ). CC/CT genotypes in rs352139 [81] were more dominant and tonsillar TLR9 expressions were significantly higher in Large $\Delta \operatorname{IgA}$ than in Small $\Delta$ IgA patients. Therefore, one can speculate that tonsillar B-cell activation via TLR9 may be involved in the production of the nephritogenic GdIgA1 in IgAN. In fact, mononuclear cells from tonsils of IgAN patients treated with CpG-DNA showed increased IgA, IFN $\gamma$, and the B-cell activating factor of the tumor necrosis factor family (BAFF) production [93]. On the other hand, Kodama et al. reported that B1 cells, which produce IgA in T-cell-independent manner, are increased in tonsils of IgAN patients [94]. Since BAFF enhanced activation of TLR in activated B1 cells [95], an increase of B1 cells may be important for pathogenesis of IgAN. In this regard, it is noteworthy that recent microarray analysis of tonsils from IgAN patients showed that tonsillar expression of APOBEC2, known as a messenger molecule for $\mathrm{B}$ cell development, is increased in IgAN [96].

Does mucosal DC expressing TLR9 play a role in the pathogenesis of IgAN? To assess whether the TLR9 on mucosal DC and B cells may play different roles in pathogenesis of IgAN, we stimulated the IgAN-prone mice with cellspecific CpG-ODN. Different CpG-ODNs have been used to study cell regulation by TLR9 in DC, and it has been shown that CpG-A-ODN induces large amounts of IFN$\alpha$ in plasmacytoid DC, that CpG-B-ODN acts as a potent stimulant of B cells, and that CpG-C-ODN functions as an activator of both B cells and DC [97-99]. Surprisingly, each CpG-ODN stimulation induced different disease phenotypes in IgAN-prone mice. Serum IgA levels increased in mice treated with CpG-B-ODN, while elevation of serum IgAIgG2a ICs was found following administration of CpG-AODN or CpG-C-ODN. The CpG-A-ODN group showed mesangial proliferation, and $\mathrm{CpG}-\mathrm{B}-\mathrm{ODN}$ therapy induced extracellular matrix expansion. The fluorescent intensity of glomerular IgA and IgG differed in each group. Those data suggest that, at least in murine IgAN, mucosal DC activation via TLR9 may link the IgA/IgG IC formation to subsequent proliferative glomerular damage.

\section{Conclusion}

As demonstrated here, clinical and experimental studies indicate that responsible $\mathrm{B}$ cells in the tonsil may link to the production of nephritogenic GdIgA1, which subsequently induces pIgA1 and IgA/IgG IC formation. The B cells seem to be activated by exogenous microbial antigens via TLR9 in mucosa and traffic systemic organs, including BM. 
Therefore, surgical removal of tonsils may directly decrease the number of the responsible $B$ cells and their activation. Additional steroid pulse therapy may thus further eliminate the responsible B cells that are systemically disseminated.

\section{Acknowledgments}

Parts of this study were supported by a Research Grant from the Study Group on IgA Nephropathy in Japan, Grantin-Aids for Progressive renal Diseases research, Research on intractable disease, from the Ministry of Health, Labor, and Welfare of Japan, and from the Ministry of Education, Culture, Sports, Science, and Technology of Japan.

\section{References}

[1] G. F. M. Strippoli, A. Maione, F. P. Schena, G. Tognoni, and J. C. Craig, "IgA nephropathy: a disease in search of a large-scale clinical trial to reliably inform practice," American Journal of Kidney Diseases, vol. 53, no. 1, pp. 5-8, 2009.

[2] J. Barratt and J. Feehally, "IgA nephropathy," Journal of the American Society of Nephrology, vol. 16, no. 7, pp. 2088-2097, 2005.

[3] J. Floege, M. Burg, and V. Kliem, "Recurrent IgA nephropathy after kidney transplantation: not a benign condition," Nephrology Dialysis Transplantation, vol. 13, no. 8, pp. 19331935, 1998.

[4] X. Cuevas, J. Lloveras, M. Mir, J. Aubia, and J. Masramon, "Disappearance of mesangial IgA deposits from the kidneys of two donors after transplantation," Transplantation Proceedings, vol. 19, no. 1, pp. 2208-2209, 1987.

[5] F. Sanfilippo, B. P. Croker, and R. R. Bollinger, "Fate of four cadaveric donor renal allografts with mesangial IgA deposits," Transplantation, vol. 33, no. 4, pp. 370-376, 1982.

[6] Y. Suzuki and Y. Tomino, "Potential immunopathogenic role of the mucosa-bone marrow axis in IgA nephropathy: insights from animal models," Seminars in Nephrology, vol. 28, no. 1, pp. 66-77, 2008.

[7] J. Barratt, J. Feehally, and A. C. Smith, "Pathogenesis of IgA nephropathy," Seminars in Nephrology, vol. 24, no. 3, pp. 197217,2004

[8] S. N. Emancipator, "Immunoregulatory factors in the pathogenesis of IgA nephropathy," Kidney International, vol. 38, no. 6, pp. 1216-1229, 1990.

[9] J. W. de Fijter, J. W. Eijgenraam, C. A. Braam et al., "Deficient IgA1 immune response to nasal cholera toxin subunit B in primary IgA nepbropathy," Kidney International, vol. 50, no. 3, pp. 952-961, 1996.

[10] J. I. Roodnat, J. W. de Fijter, C. van Kooten, M. R. Daha, and L. A. Van Es, "Decreased IgAl response after primary oral immunization with live typhoid vaccine in primary IgA nephropathy," Nephrology Dialysis Transplantation, vol. 14, no. 2, pp. 353-359, 1999.

[11] Y. Xie, S. Nishi, M. Ueno et al., "The efficacy of tonsillectomy on long-term renal survival in patients with IgA nephropathy," Kidney International, vol. 63, no. 5, pp. 1861-1867, 2003.

[12] M. Sato, O. Hotta, S. Tomioka et al., "Cohort study of advanced IgA nephropathy: efficacy and limitations of corticosteroids with tonsillectomy," Nephron Clinical Practice, vol. 93, no. 4, pp. 137-145, 2003.

[13] O. Hotta, M. Miyazaki, T. Furuta et al., "Tonsillectomy and steroid pulse therapy significantly impact on clinical remission in patients with IgA nephropathy," American Journal of Kidney Diseases, vol. 38, no. 4, pp. 736-743, 2001.
[14] H. Komatsu, S. Fujimoto, S. Hara, Y. Sato, K. Yamada, and K. Kitamura, "Effect of tonsillectomy plus steroid pulse therapy on clinical remission of IgA nephropathy: a controlled study," Clinical Journal of the American Society of Nephrology, vol. 3, no. 5, pp. 1301-1307, 2008.

[15] F. M. Rasche, A. Schwarz, and F. Keller, "Tonsillectomy does not prevent a progressive course in IgA nephropathy," Clinical Nephrology, vol. 51, no. 3, pp. 147-152, 1999.

[16] A. Piccoli, M. Codognotto, M. G. Tabbi, E. Favaro, and B. Rossi, "Influence of tonsillectomy on the progression of mesangioproliferative glomerulonephritis," Nephrology Dialysis Transplantation, vol. 25, no. 8, pp. 2583-2589, 2010.

[17] Y. Chen, Z. Tang, Q. Wang et al., "Long-term efficacy of tonsillectomy in Chinese patients with IgA nephropathy," American Journal of Nephrology, vol. 27, no. 2, pp. 170-175, 2007.

[18] L. S. Ibels, A. Z. Gyory, R. J. Caterson et al., "Recognition and management of IgA nephropathy," Drugs, vol. 55, no. 1, pp. 73-83, 1998.

[19] N. Miura, H. Imai, S. Kikuchi et al., “Tonsillectomy and steroid pulse (TSP) therapy for patients with IgA nephropathy: a nationwide survey of TSP therapy in Japan and an analysis of the predictive factors for resistance to TSP therapy," Clinical and Experimental Nephrology, vol. 13, no. 5, pp. 460-466, 2009.

[20] A. Kataura and H. Tsubota, "Clinical analyses of focus tonsil and related diseases in Japan," Acta Oto-Laryngologica, no. 523, supplement, pp. 161-164, 1996.

[21] T. Ono, M. Jono, M. Kito et al., "Evaluation of tonsillectomy as a treatment for pustulosis palmaris et plantaris," Acta Oto-Laryngologica, vol. 401, pp. 12-16, 1983.

[22] M. Yokoyama, K. Hashiguchi, and Y. Yamasaki, "Effects of tonsillectomy in patients with pusutulosis Palmaris et plantaris," Acta Oto-Laryngologica, vol. 124, pp. 1109-1110, 2004.

[23] K. Noda, S. Kodama, S. Suenaga, and M. Suzuki, "Tonsillar focal infectious disease involving IgA nephropathy, pustulosis, and ossification," Clinical and Experimental Nephrology, vol. 11, no. 1, pp. 97-101, 2007.

[24] Y. Tomino, M. Endoh, Y. Nomoto, and H. Sakai, "Immunoglobulin A1 and IgA nephropathy," New England Journal of Medicine, vol. 305, no. 19, pp. 1159-1160, 1981.

[25] A. W. L. van den Wall Bake, J. Evers-Schouten, and L. A. van Es, "Serum IgA and the production of IgA by peripheral blood and bone marrow lymphocytes in patients with primary IgA nephropathy: evidence for the bone marrow as the source of mesangial IgA," American Journal of Kidney Diseases, vol. 12, no. 5, pp. 410-414, 1988.

[26] O. Sakai, "IgA nephropathy: current concepts and feature trends," Nephrology, vol. 3, pp. 2-3, 1997.

[27] Y. Iwata, T. Wada, A. Uchiyama et al., "Remission of IgA nephropathy after allogeneic peripheral blood stem cell transplantation followed by immunosuppression for acute lymphocytic leukemia," Internal Medicine, vol. 45, no. 22, pp. 1291-1295, 2006.

[28] L. Layward, A. M. Finnemore, A. C. Allen, S. J. Harper, and J. Feehally, "Systemic and mucosal IgA responses to systemic antigen challenge in IgA nephropathy," Clinical Immunology and Immunopathology, vol. 69, no. 3, pp. 306-313, 1993.

[29] A. W. van den Wall Bake, W. E. Beyer, J. H. Evers-Schouten et al., "Humoral immune response to influenza vaccination in patients with primary immunoglobulin A nephropathy. An analysis of isotype distribution and size of the influenzaspecific antibodies," Journal of Clinical Investigation, vol. 84, no. 4, pp. 1070-1075, 1989. 
[30] M. C. Bene, G. Faure, B. H. de Ligny et al., "Immunoglobulin A nephropathy. Quantitative immunohistomorphometry of the tonsillar plasma cells evidences an inversion of the immunoglobulin A versus immunoglobulin G secreting cell balance," Journal of Clinical Investigation, vol. 71, no. 5, pp. 1342-1347, 1983.

[31] S. J. Harper and J. Feehally, "The pathogenic role of immunoglobulin A polymers in immunoglobulin A nephropathy," Nephron, vol. 65, no. 3, pp. 337-345, 1993.

[32] C. Kusakari, M. Nose, T. Takasaka et al., "Immunopathological features of palatine tonsil characteristic of IgA nephropathy: IgA1 localization in follicular dendritic cells," Clinical and Experimental Immunology, vol. 95, no. 1, pp. 42-48, 1994.

[33] S. J. Harper, J. H. Pringle, A. C. B. Wicks et al., "Expression of J chain mRNA in duodenal IgA plasma cells in IgA nephropathy," Kidney International, vol. 45, no. 3, pp. 836-844, 1994.

[34] A. W. van den Wall Bake, M. R. Daha, and L. A. van Es, "Immunopathogenetic aspects of IgA nephropathy," Nephrologie, vol. 10, no. 3, pp. 141-145, 1989.

[35] L. A. van Es, A. W. van den Wall Bake, R. K. Stad, M. E. van den Dobbelsteen, M. J. Bogers, and M. R. Daha, "Enigmas in the pathogenesis of IgA nephropathy," Contributions to Nephrology, vol. 111, pp. 169-175, 1995.

[36] E. J. Kunkel and E. C. Butcher, "Plasma-cell homing," Nature Reviews Immunology, vol. 3, no. 10, pp. 822-829, 2003.

[37] Z. Moldoveanu, R. J. Wyatt, J. Y. Lee et al., "Patients with IgA nephropathy have increased serum galactose-deficient IgA1 levels," Kidney International, vol. 71, no. 11, pp. 1148-1154, 2007.

[38] H. Suzuki, Z. Moldoveanu, S. Hall et al., "IgA1-secreting cell lines from patients with IgA nephropathy produce aberrantly glycosylated IgA1," Journal of Clinical Investigation, vol. 118, no. 2, pp. 629-639, 2008.

[39] R. J. Glassock, "Analyzing antibody activity in IgA nephropathy," Journal of Clinical Investigation, vol. 119, no. 6, pp. 1450-1452, 2009.

[40] Y. Hiki, H. Odani, M. Takahashi et al., "Mass spectrometry proves under-O-glycosylation of glomerular IgA1 in IgA nephropathy," Kidney International, vol. 59, no. 3, pp. 1077-1085, 2001.

[41] A. C. Allen, E. M. Bailey, P. E. C. Brenchley, K. S. Buck, J. Barratt, and J. Feehally, "Mesangial Igal in IgA nephropathy exhibits aberrant $O$-glycosylation: observations in three patients," Kidney International, vol. 60, no. 3, pp. 969-973, 2001.

[42] T. Kokubo, Y. Hiki, H. Iwase et al., "Protective role of IgA1 glycans against IgA1 self- aggregation and adhesion to extracellular matrix proteins," Journal of the American Society of Nephrology, vol. 9, no. 11, pp. 2048-2054, 1998.

[43] J. Novak, B. A. Julian, M. Tomana, and J. Mestecky, "IgA glycosylation and IgA immune complexes in the pathogenesis of IgA nephropathy," Seminars in Nephrology, vol. 28, no. 1, pp. 78-87, 2008.

[44] Y. Hiki, "O-linked oligosaccharides of the IgA1 hinge region: roles of its aberrant structure in the occurrence and/or progression of IgA nephropathy," Clinical and Experimental Nephrology, vol. 13, no. 5, pp. 415-423, 2009.

[45] J. Novak, M. Tomana, K. Matousovic et al., "IgA1-containing immune complexes in IgA nephropathy differentially affect proliferation of mesangial cells," Kidney International, vol. 67, no. 2, pp. 504-513, 2005.

[46] J. Novak, H. L. Vu, L. Novak, B. A. Julian, J. Mestecky, and M. Tomana, "Interactions of human mesangial cells with IgA and IgA-containing immune complexes," Kidney International, vol. 62 , no. 2, pp. 465-475, 2002.
[47] C. Gómez-Guerrero, E. González, and J. Egido, "Evidence for a specific IgA receptor in rat and human mesangial cells," Journal of Immunology, vol. 151, no. 12, pp. 7172-7181, 1993.

[48] C. Gómez-Guerrero, M. J. López-Armada, E. González, and J. Egido, "Soluble IgA and IgG aggregates are catabolized by cultured rat mesangial cells and induce production of TNF- $\alpha$ and IL-6, and proliferation," Journal of Immunology, vol. 153, no. 11, pp. 5247-5256, 1994.

[49] P. J. M. van der Boog, C. van Kooten, J. W. de Fijter, and M. R. Daha, "Role of macromolecular IgA in IgA nephropathy," Kidney International, vol. 67, no. 3, pp. 813-821, 2005.

[50] J. Mestecky, M. Tomana, P. A. Crowley-Nowick, Z. Moldoveanu, B. A. Julian, and S. Jackson, "Defective galactosylation and clearance of IgA1 molecules as a possible etiopathogenic factor in IgA nephropathy," Contributions to Nephrology, vol. 104, pp. 172-182, 1993.

[51] R. Coppo and A. Amore, "Aberrant glycosylation in IgA nephropathy (IgAN)," Kidney International, vol. 65, no. 5, pp. 1544-1547, 2004.

[52] A. Horie, Y. Hiki, H. Odani et al., "IgA1 molecules produced by tonsillar lymphocytes are under-O-glycosylated in IgA nephropathy," American Journal of Kidney Diseases, vol. 42, no. 3, pp. 486-496, 2003.

[53] A. Itoh, H. Iwase, T. Takatani et al., "Tonsillar IgA1 as a possible source of hypoglycosylated IgA1 in the serum of IgA nephropathy patients," Nephrology Dialysis Transplantation, vol. 18, no. 6, pp. 1108-1114, 2003.

[54] N. Iwanami, H. Iwase, N. Takahashi et al., "Similarities between $\mathrm{N}$-glycan glycoform of tonsillar IgAl and that of aberrant IgA1 abundant in IgA nephropathy patient serum," Journal of Nephrology, vol. 21, no. 1, pp. 118-126, 2008.

[55] T. Inoue, H. Sugiyama, Y. Hiki et al., "Differential expression of glycogenes in tonsillar B lymphocytes in association with proteinuria and renal dysfunction in IgA nephropathy," Clinical Immunology, vol. 136, no. 3, pp. 447-455, 2010.

[56] R. C. Monteiro and J. G. J. Van De Winkel, "IgA Fc receptors," Annual Review of Immunology, vol. 21, pp. 177-204, 2003.

[57] T. Nishie, O. Miyaishi, H. Azuma et al., "Development of immunoglobulin A nephropathy-like disease in $\beta-1,4$ galactosyltransferase-I-deficient mice," American Journal of Pathology, vol. 170, no. 2, pp. 447-456, 2007.

[58] I. Kobayashi, F. Nogaki, H. Kusano et al., "Interleukin-12 alters the physicochemical characteristics of serum and glomerular IgA and modifies glycosylation in a ddY mouse strain having high IgA levels," Nephrology Dialysis Transplantation, vol. 17, no. 12, pp. 2108-2116, 2002.

[59] R. J. Glassock, "IgA nephropathy: challenges and opportunities," Cleveland Clinic Journal of Medicine, vol. 75, no. 8, pp. 569-576, 2008.

[60] A. G. Gharavi, Z. Moldoveanu, R. J. Wyatt et al., "Aberrant IgA1 glycosylation is inherited in familial and sporadic IgA nephropathy," Journal of the American Society of Nephrology, vol. 19, no. 5, pp. 1008-1014, 2008.

[61] H. Suzuki, R. Fan, Z. Zhang et al., "Aberrantly glycosylated $\operatorname{IgA1}$ in IgA nephropathy patients is recognized by IgG antibodies with restricted heterogeneity," Journal of Clinical Investigation, vol. 119, no. 6, pp. 1668-1677, 2009.

[62] J. Barratt, F. Eitner, J. Feehally, and J. Floege, "Immune complex formation in IgA nephropathy: a case of the 'right' antibodies in the 'wrong' place at the 'wrong' time," Nephrology Dialysis Transplantation, vol. 24, no. 12, pp. 3620-3623, 2009.

[63] J. Barratt and F. Eitner, "Glomerular disease: sugars and immune complex formation in IgA nephropathy," Nature Reviews Nephrology, vol. 5, pp. 612-614, 2009. 
[64] H. Suzuki, Y. Suzuki, M. Aizawa et al., "Th1 polarization in murine IgA nephropathy directed by bone marrow-derived cells," Kidney International, vol. 72, no. 3, pp. 319-327, 2007.

[65] P. O. Leinikki, J. Mustonen, and A. Pasternack, "Immune response to oral polio vaccine in patients with IgA glomerulonephritis," Clinical and Experimental Immunology, vol. 68, no. 1, pp. 33-38, 1987.

[66] S. N. Emancipator, G. R. Gallo, and M. E. Lamm, "Experimental IgA nephropathy induced by oral immunization," Journal of Experimental Medicine, vol. 157, no. 2, pp. 572-582, 1983.

[67] R. Coppo, B. Basolo, and C. Rollino, "Mediterranean diet and primary IgA nephropathy," Clinical Nephrology, vol. 26, no. 2, pp. 72-82, 1986.

[68] V. Kumar, M. Sieniawska, E. H. Beutner, and T. P. Chorzelski, "Are immunological markers of gluten-sensitive enteropathy detectable in IgA nephropathy?" The Lancet, vol. 2, no. 8623, p. 1307, 1988.

[69] A. Fornasieri, R. A. Sinico, P. Maldifassi et al., "IgA-Antigliadin antibodies in IgA mesangial nephropathy (Berger's disease)," British Medical Journal, vol. 295, no. 6590, pp. 78-80, 1987.

[70] G. Rostoker, J. Laurent, C. André, S. Cholin, and G. Lagrue, "High levels of IgA antigliadin antibodies in patients who have IgA mesangial glomerulonephritis but not coeliac disease," The Lancet, vol. 1, no. 8581, pp. 356-357, 1988.

[71] R. Coppo, G. Mazzucco, G. Martina et al., "Gluten-induced experimental IgA glomerulopathy," Laboratory Investigation, vol. 60 , no. 4, pp. 499-506, 1989.

[72] D. Yan, W. K. Rumbeiha, and J. J. Pestka, "Experimental murine IgA nephropathy following passive administration of vomitoxin-induced IgA monoclonal antibodies," Food and Chemical Toxicology, vol. 36, no. 12, pp. 1095-1106, 1998.

[73] J. J. Pestka, "Deoxynivalenol-induced IgA production and IgA nephropathy-aberrant mucosal immune response with systemic repercussions," Toxicology Letters, vol. 140-141, pp. 287-295, 2003.

[74] Y. Shi and J. J. Pestka, "Attenuation of mycotoxin-induced IgA nephropathy by eicosapentaenoic acid in the mouse: dose response and relation to IL-6 expression," Journal of Nutritional Biochemistry, vol. 17, no. 10, pp. 697-706, 2006.

[75] F. Hinoshita, Y. Suzuki, K. Yokoyama et al., "Experimental IgA nephropathy induced by a low-dose environmental mycotoxin, nivalenol," Nephron, vol. 75, no. 4, pp. 469-478, 1997.

[76] H. K. Smerud, B. Fellström, R. Hällgren, S. Osagie, P. Venge, and G. Kristjánsson, "Gastrointestinal sensitivity to soy and milk proteins in patients with IgA nephropathy," Clinical Nephrology, vol. 74, no. 5, pp. 364-371, 2010.

[77] A. C. Smith, K. Molyneux, J. Feehally, and J. Barratt, "Oglycosylation of serum IgA1 antibodies against mucosal and systemic antigens in IgA nephropathy," Journal of the American Society of Nephrology, vol. 17, no. 12, pp. 3520-3528, 2006.

[78] H. Suzuki, Y. Suzuki, T. Yamanaka et al., "Genome-wide scan in a novel IgA nephropathy model identifies a susceptibility locus on murine chromosome 10 , in a region syntenic to human IGAN1 on chromosome 6q22-23," Journal of the American Society of Nephrology, vol. 16, no. 5, pp. 1289-1299, 2005.

[79] S. Akira, K. Takeda, and T. Kaisho, "Toll-like receptors: critical proteins linking innate and acquired immunity," Nature Immunology, vol. 2, no. 8, pp. 675-680, 2001.

[80] S. Akira and K. Takeda, "Toll-like receptor signalling," Nature Reviews Immunology, vol. 4, no. 7, pp. 499-511, 2004.

[81] H. Suzuki, Y. Suzuki, I. Narita et al., "Toll-like receptor 9 affects severity of IgA nephropathy," Journal of the American Society of Nephrology, vol. 19, no. 12, pp. 2384-2395, 2008.
[82] T. Kaisho and S. Akira, "Dendritic-cell function in Toll-like receptor- and MyD88-knockout mice," Trends in Immunology, vol. 22, no. 2, pp. 78-83, 2001.

[83] A. A. Ashkar and K. L. Rosenthal, "Toll-like receptor 9, CpG DNA and innate immunity," Current Molecular Medicine, vol. 2, no. 6, pp. 545-556, 2002.

[84] E. A. Leadbetter, I. R. Rifkin, and A. Marshak-Rothstein, "Toll-like receptors and activation of autoreactive B cells," Current Directions in Autoimmunity, vol. 6, pp. 105-122, 2003.

[85] R. H. Carter and R. Myers, "Germinal center structure and function: lessons from CD19," Seminars in Immunology, vol. 20, no. 1, pp. 43-48, 2008.

[86] R. D. Sanderson, P. Lalor, and M. Bernfield, "B lymphocytes express and lose syndecan at specific stages of differentiation," Cell Regulation, vol. 1, no. 1, pp. 27-35, 1989.

[87] M. Aizawa, Y. Suzuki, H. Suzuki et al., "Roles of bone marrow, mucosa and lymphoid tissues in pathogenesis of murine IgA nephropathy," Contributions to Nephrology, vol. 157, pp. 164-168, 2007.

[88] R. Shinkura, F. Matsuda, T. Sakiyama et al., "Defects of somatic hypermutation and class switching in alymphoplasia (aly) mutant mice," International Immunology, vol. 8, no. 7, pp. 1067-1075, 1996.

[89] Y. Masuda, S. Tamura, and N. Sugiyama, "The effect of tonsillectomy and its postoperative clinical course in IgA nephropathy with chronic tonsillitis," Advances in Oto-RhinoLaryngology, vol. 47, pp. 203-207, 1992.

[90] Y. Masuda, K. Terazawa, S. Kawakami, Y. Ogura, and N. Sugiyama, "Clinical and immunological study of IgA nephropathy before and after tonsillectomy," Acta Oto-Laryngologica, vol. 106, no. 454, supplement, pp. 248-255, 1988.

[91] S. Tomioka, K. Miyoshi, K. Tabata, O. Hotta, and Y. Taguma, "Clinical study of chronic tonsillitis with IgA nephropathy treated by tonsillectomy," Acta Oto-Laryngologica, no. 523, supplement, pp. 175-177, 1996.

[92] D. Sato, Y. Suzuki, T. Kano et al., “Tonsillar TLR9 expression and efficacy of tonsillectomy with steroid pulse therapy in IgA nephropathy patients," Nephrology Dialysis Transplantation, 2011. In press.

[93] T. Goto, N. Bandoh, T. Yoshizaki et al., "Increase in B-cell-activation factor (BAFF) and IFN- $\gamma$ productions by tonsillar mononuclear cells stimulated with deoxycytidyl-deoxyguanosine oligodeoxynucleotides (CpG-ODN) in patients with IgA nephropathy," Clinical Immunology, vol. 126, no. 3, pp. 260-269, 2008.

[94] S. Kodama, M. Suzuki, M. Arita, and G. Mogi, "Increase in tonsillar germinal centre B-1 cell numbers in IgA nephropathy (IgAN) patients and reduced susceptibility to Fas-mediated apoptosis," Clinical and Experimental Immunology, vol. 123, no. 2, pp. 301-308, 2001.

[95] L. G. Ng, C. H. Ng, B. Woehl et al., "BAFF costimulation of Toll-like receptor-activated B-1 cells," European Journal of Immunology, vol. 36, no. 7, pp. 1837-1846, 2006.

[96] K. Iio, Y. Nagasawa, H. Iwatani et al., "Microarray analysis of tonsils in immunoglobulin A nephropathy patients," Biochemical and Biophysical Research Communications, vol. 393, no. 4, pp. 565-570, 2010.

[97] A. Krug, A. Towarowski, S. Britsch et al., "Toll-like receptor expression reveals CpG DNA as a unique microbial stimulus for plasmacytoid dendritic cells which synergizes with $\mathrm{Cd} 40$ ligand to induce high amounts of IL-12," European Journal of Immunology, vol. 31, no. 10, pp. 3026-3037, 2001. 
[98] H. Hemmi, T. Kaisho, O. Takeuchi et al., "Small-antiviral compounds activate immune cells via the TLR7 MyD88dependent signaling pathway," Nature Immunology, vol. 3, no. 2, pp. 196-200, 2002.

[99] M. Kerkmann, S. Rothenfusser, V. Hornung et al., "Activation with CpG-A and CpG-B oligonucleotides reveals two distinct regulatory pathways of type I IFN synthesis in human plasmacytoid dendritic cells," Journal of Immunology, vol. 170, no. 9, pp. 4465-4474, 2003. 


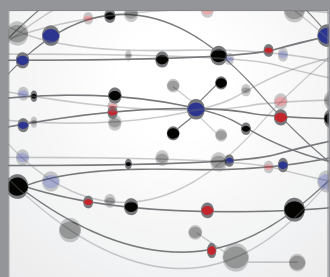

The Scientific World Journal
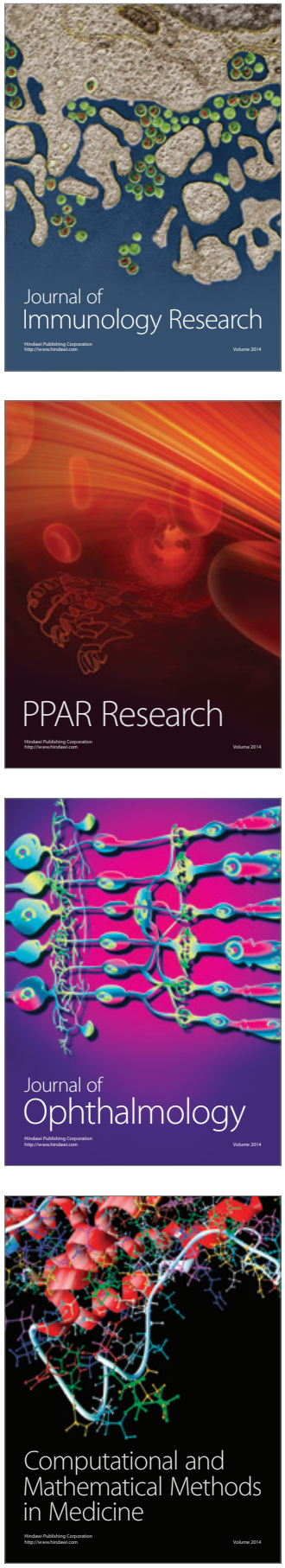

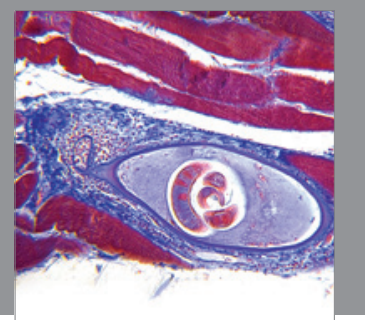

Gastroenterology

Research and Practice
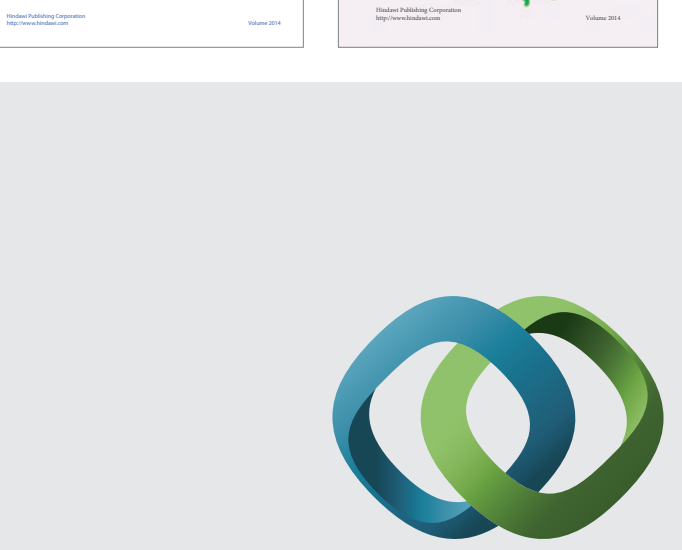

\section{Hindawi}

Submit your manuscripts at

http://www.hindawi.com
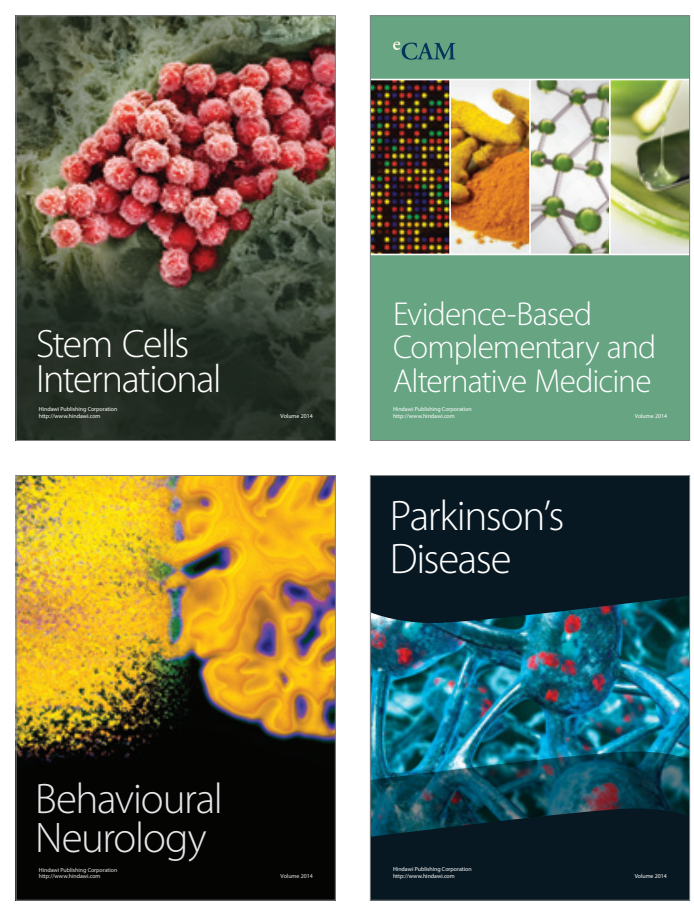

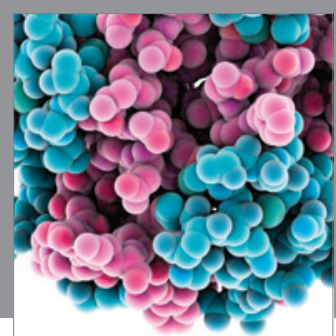

Journal of
Diabetes Research

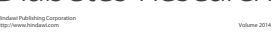

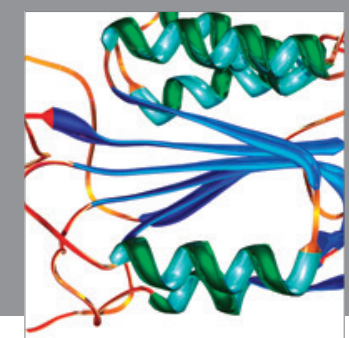

Disease Markers
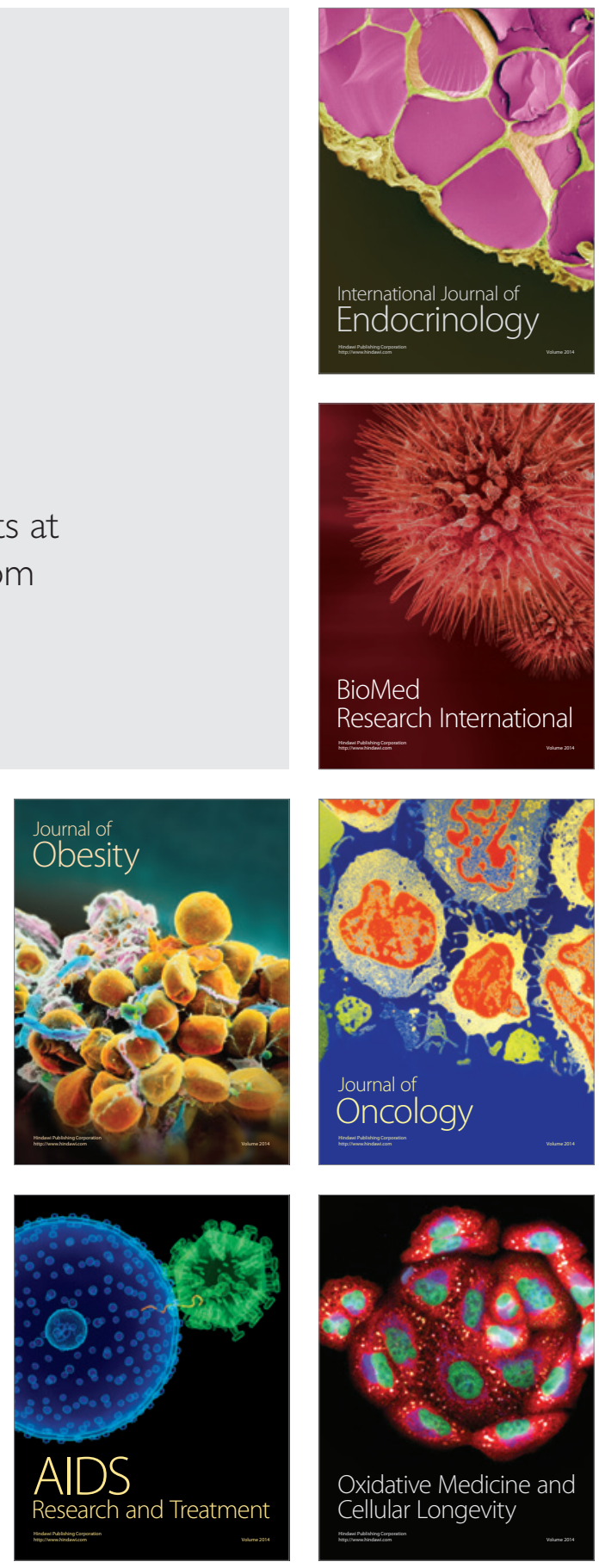